\title{
Féeries
}

Études sur le conte merveilleuX, XVII ${ }^{e}$ XIXe siècle

$9 \mid 2012$

Le dialogisme intertextuel des contes des Grimm

\section{Persinette en Allemagne : le corpus « purement allemand pour sa naissance et sa mise en forme " des frères Grimm}

Persinette in Germany: the "exclusively german corpus in its origin and shape" by the brothers Grimm

\section{Jean Mainil}

\section{CpenEdition \\ Journals}

Édition électronique

URL : http://journals.openedition.org/feeries/817

ISSN : 1957-7753

Éditeur

UGA Éditions/Université Grenoble Alpes

Édition imprimée

Date de publication : 15 octobre 2012

Pagination : 29-54

ISBN : 978-2-84310-233-2

ISSN : 1766-2842

Référence électronique

Jean Mainil, «Persinette en Allemagne : le corpus « purement allemand pour sa naissance et sa mise en forme » des frères Grimm », Féeries [En ligne], 9 | 2012, mis en ligne le 15 octobre 2013, consulté le 07 septembre 2020. URL : http://journals.openedition.org/feeries/817 


\author{
Jean Mainil \\ Universiteit Gent (UGent), Belgique
}

PERSINETTE EN ALLEMAGNE :

LE CORPUS «PUREMENT ALLEMAND POUR SA NAISSANCE

ET SA MISE EN FORME» DES FRÈRES GRIMM

$D$

ANS LE DOMAINE des études sur le conte de fées, l'analyse des intertextes et des influences - que celles-ci soient possibles ou probables - est souvent un sujet épineux pour trois raisons qui relèvent de la nature du conte, des versions antérieures connues, ainsi que de la poétique des conteurs et conteuses. Je développerai d'abord ces raisons pour ensuite concentrer mon analyse sur trois contes qui entretiennent des liens intertextuels indéniables. Il ne s'agit pas de retracer ici un putatif "réseau d'influences", mais plutôt d'analyser un cas précis de dialogues intertextuels qu'entretiennent trois textes identifiés à une même catégorie générique, le conte de fées. Jacob et Wilhelm Grimm ont donné une version de ce conte dans le premier tome des Kinder-und Hausmärchen dont nous fêtons le bicentenaire : Rapunzel.

\title{
Le conte dialogique... un dialogue, mais avec qui?
}

Comme Raymonde Robert l'a rappelé récemment :

Les contes d'origine populaire ont ceci de particulier qu'ils appartiennent à un patrimoine qui dépasse largement toutes les frontières, qu'elles soient géographiques ou linguistiques. Comme ils n'ont pas d'auteurs repérables, les chercheurs sont encore réduits, à l'époque actuelle, à échafauder des hypothèses pour expliquer où, et quand et comment ils ont été produits. C'est dire que prétendre étudier des textes écrits qui s'en inspirent est une entreprise difficile et parfois aléatoire ${ }^{2}$.

I. Mes plus vifs remerciements vont à U. Heidmann pour ses lectures et son amitié, mais aussi pour avoir partagé son expertise en napolitain et en allemand pour les traductions de G. Basile et des Grimm.

2. R. Robert, «Les Conteurs français lecteurs de Basile», 2008, p. 333. 
D'une plasticité qui résiste à tous les siècles, à toutes les traductions d'une langue à une autre, d'un contexte idéologique ou politique à un autre, le conte a survécu, mais souvent de manière anonyme. Non seulement, comme l'a rappelé $\mathrm{R}$. Robert, l'origine du conte reste parfois inconnue, mais même quand elle ne l'est pas - dans le cas de contes écrits et littéraires - le mode de transmission conduit également à d'autres incertitudes quant à son origine.

Le conte a, contrairement aux autres genres modernes, ceci de particulier qu'il est resté un genre à la fois oral et écrit et qu'il n'existe pas de frontière étanche entre les deux modes de transmission. Est-ce le prix à payer pour son étonnante plasticité et son universalité? Le conte de fées est souvent resté anonyme, les traces qu'il laisse sont souvent vagues, impersonnelles, même lorsque des versions bien précises sont attestées et clairement identifiées à un auteur. On peut ainsi appliquer au conte en général ce que Marc Escola a déclaré au sujet des Contes de Perrault : «Les Contes de Perrault jouissent d'un privilège assez rare : on les connaît sans avoir à les lire ${ }^{3}$." Le conte de fées fait en effet partie de ce que Jan Herman a appelé la «bibliothèque culturelle, l'encyclopédie intérieure, du lecteur». Le conte de fées pourrait à ce titre fonctionner comme une situation extrême, voire exemplaire, de ce qu'Herman qualifie de "topique» : "une situation narrative récurrente reconnue comme telle par une collectivité de lecteurs ${ }^{4}$.

Dans le cas des infinies reconfigurations génériques du conte, cette situation topique est d'autant plus universelle qu' elle ne se limite pas à l'écrit et qu'elle s'étend également aux domaines de l'oral et du visuel. Au XIX ${ }^{\mathrm{e}}$ siècle, les illustrations, immortalisées par celles de Gustave Doré, se font de plus en plus présentes dans toutes les éditions, permettant des élaborations narratives (Lhéritier aurait dit «broderies») à partir de ces visualisations précises. Mais à partir du milieu du $\mathrm{xx}^{\mathrm{e}}$ siècle, ce sera Walt Disney qui fera finalement régner le visuel sur le domaine du merveilleux. Alors, quand on connaît aujourd'hui un conte, comment se souvenir d'où nous l'avons appris : l'avons-nous lu, l'avons-nous entendu, si oui, de quelqu'un qui l'aurait lu, entendu, ou vu, ou l'aurions-nous vu?

S'il appartient à «l'encyclopédie universelle» de tout un chacun, le conte de fées a subi également des variations et des transformations plus ou moins profondes dans le volume virtuel, mental et imaginaire que chacun de nous possède de cette encyclopédie. Atemporel, oral mais aussi écrit, le conte de fées est à la fois universel et personnel. Qui a enseigné Le Petit Chaperon

3. M. Escola, Marc Escola commente les Contes de Charles Perrault, 2005, p. II.

4. J. Herman, M. Kozul et N. Kremer, Le Roman véritable, 2008, p. Io8. 
rouge sait que, quand on parle de ce conte de Charles Perrault, on parle en réalité de la version des frères Grimm. Quand on parle de La Belle au bois dormant, de Charles Perrault encore, on parle en réalité de La Belle au bois dormant des studios Disney. Non seulement, on a atrophié le conte de sa seconde moitié, mais le prince doit encore combattre vaillamment les éléments hostiles pour atteindre la belle Princesse endormie, alors que chez le conteur français, ces éléments hostiles ne le sont pas pour le prince. Quant au réveil de la Princesse, la scène romantique de Disney où c'est le baiser du Prince qui réveille la Princesse a, à jamais, remplacé la scène ironique chez Perrault où la Princesse se réveille simplement parce que les Ioo ans se sont écoulés'. Quand on parle de la Cendrillon de Perrault, c'est celle de Disney que l'on voit : elle est la parfaite victime silencieuse des pires avanies de sa belle-mère et de ses demi-sœurs. On a oublié l'hypocrisie manipulatrice de l'héroïne de Perrault qui attend patiemment le retour de ses demi-sœurs pour que celles-ci lui parlent de cette magnifique princesse inconnue qui est arrivée au bal et qui n'est autre qu'elle-même. On a oublié aussi que la même Cendrillon, sûre d'un refus, demande à l'une de ses sœurs de lui prêter une robe, provoquant la colère et la moquerie de celle-ci et s'assurant ainsi d'un habit magique qu' elle obtient à force de pleurs qui finissent par attendrir la bienveillante marraine. Il en va des contes comme du reste, nous avons la mémoire bien sélective. Universel, le conte de fées est aussi essentiellement individuel, même quand nous avons lu, entendu, ou vu, une même version.

On pourrait a priori croire que l'existence de versions écrites antérieures puisse entraîner plus de certitudes quant aux modalités de reconfigurations génériques précises de contes. Mais ce n'est pas le cas non plus. Comme l'a encore rappelé R. Robert dans l'article déjà cité, deux sources possibles des contes de fées écrits aux XVII ${ }^{\mathrm{e}}$ et XVIII ${ }^{\mathrm{e}}$ siècles, les Piacevoli Notti de Giovan Francesco Straparola (I550-1553) ${ }^{6}$ et le recueil de Giambattista Basile, Lo cunto de li cunti $(\mathrm{I} 634)^{7}$, soulèvent le problème de la connaissance que pouvaient en avoir les conteurs et conteuses de l'Ancien Régime. Si les Piacevoli Notti avaient été traduites dès I573, le recueil de Basile, en dialecte napolitain, ne sera pas traduit en français au XVII ${ }^{\mathrm{e}}$ siècle, mais en italien, plus facile d'accès ${ }^{8}$. Mais même la possibilité de lire un recueil de contes ne

5. Voir au sujet de l'ironie de cette scène aussi U. Heidmann, «Expérimention générique et dialogisme intertextuel», 20II, p. 52-62.

6. Traduites en français sous le titre des Nuits Facétieuses.

7. Traduit en français sous le titre du Conte des contes ou Le Divertissement des petits enfants.

8. Voir l'analyse de R. Robert, art. cité, p. 333-334. 
peut rien garantir. Une transmission orale d'un conte lu donne encore une autre version de ce même conte.

À l'absence de souvenir précis qui ne nous permet plus pour des raisons diverses (trop jeunes pour se souvenir, sources indéfinies, lecture, conte oral, conte entendu, conte vu...) d'identifier précisément une source, à l'incertitude qui frappe la possibilité de lire un conte à une époque s'ajoute encore une dernière raison que j'appellerai l'amnésie sélective des conteurs et des conteuses. Ces raisons ne tiennent plus du mode de transmission, ni de la possibilité de lire ou d'entendre des contes à une époque : elles sont à la fois poétiques et idéologiques.

Dans le domaine de la littérature, le conte de fées n'échappe pas à une tendance générale par laquelle nombre d'auteurs prennent par rapport à la production littéraire antérieure une distance stratégique qui leur permet de clamer haut et fort leur propre nouveauté, leur propre modernité. Dans une apostrophe «Au lecteur» contemporaine des premiers contes de fées français, Eustache Le Noble déclarait en I694 dans Ildegerte, Reine de Norvège, ou l'Amour Magnanime:

Les goûts sur les livres changent de mode chez les Français comme les habits. Les longs romans pleins de paroles et d'aventures fabuleuses, et vides des choses qui doivent rester dans l'esprit du lecteur et y faire du fruit, étaient en vogue dans le temps que les chapeaux pointus étaient trouvés beaux. On s'est lassé presque en même temps des uns et des autres, et les petites histoires ornées des agréments que la vérité peut souffrir ont pris leur place et se sont trouvées plus propres au génie français, qui est impatient de voir en deux heures le dénouement et la fin de ce qu'il commence à lire?

En 1694, Eustache Le Noble ne pouvait pas prévoir qu'un genre nouveau supplanterait bientôt le chapeau nouveau en allant dans le même sens, mais beaucoup plus radicalement. Si Ildegerte s'oppose aux longs romans, elle comporte quand même I24 pages pour sa première partie, suivie d'une seconde de II9, soit 243 pages. Certes, certains contes encadrés de MarieCatherine d'Aulnoy seront encore nettement plus longs, mais beaucoup d'autres contes seront aussi nettement plus brefs. Et ils supplanteront en effet les Ildegerte et autres nouvelles historiques au profit de ce genre court - ou séparable, comme le pratiqueront les éditeurs de contes encadrés au XIX ${ }^{\mathrm{e}}$ siècle qui les publieront littéralement hors contexte — qui, trois années après l'histoire de Le Noble, envahira les presses parisiennes et fera les délices du public.

9. E. Le Noble, «Au lecteur», Ildegerte, n. p. 
À certains moments historiques précis, cette démarcation peut prendre une dimension poétique, comme ce fut le cas pour le premier conte de fées littéraire, véritable emblème des Modernes dans la Querelle qui les opposait aux Anciens. À d'autres moments historiques précis, cette mise à distance du corpus antérieur peut aussi être à la fois poétique et politique, quand elle prend une dimension nationaliste par exemple. C'était déjà le cas du "génie national » de Le Noble il y a trois siècles, ce sera aussi le cas il y a deux siècles en Allemagne et plus tard dans le siècle, en France, à partir des années I860. Un nouveau corpus basé sur une poétique nouvelle s'inscrit ainsi, au XIX ${ }^{\mathrm{e}}$ siècle, dans la production d'un corpus national qui s'invente et reflète une identité nationale à laquelle, en réalité, elle contribue. La récupération du conte et sa transformation en «artefacts culturels d'un type bien particulier» qui contribuent à un "imaginaire national ${ }^{\mathrm{IO}}$ » naissant sont d'autant plus délicates et particulières que le corpus générique du conte est, par essence, international et intertextuel.

\section{Un dialogue entre trois contes: Petrosinella, Persinette et Rapunzel ${ }^{\mathrm{II}}$}

Nous considérerons à présent un conte tiré du premier tome des Kinderund Hausmärchen dans le cadre de l'entreprise poétique et politique que proposaient Jacob et Wilhelm Grimm il y a deux siècles exactement. Ce conte, l'un des plus connus, Rapunzel, traduit en français sous le nom de Raiponce, entretient un dialogue avec les domaines napolitain (Basile) et français (Charlotte-Rose de Caumont de La Force et Marie-Catherine d'Aulnoy). Nous analyserons les éléments considérés comme constitutifs du conte pour essayer de mieux rendre compte des imbrications orales et écrites, et donc des réseaux intertexuels génériques, qui informent la production du conte de fées des frères Grimm ${ }^{12}$.

Nous proposons ici une lecture, côte à côte, de ces contes qui entretiennent tous des liens étroits et trouvent, dans le premier volume des

Io. B. Anderson, L'imaginaire national, 1996.

II. Chatte Blanche de M.-C. d'Aulnoy inclut en deuxième partie le conte 310, Persinette. Cette combinaison en séquence du conte à un autre conte, son encadrement dans une nouvelle, «Le Nouveau Gentilhomme bourgeois. Conte», ainsi que son lieu d'énonciation (son attribution à une conteuse intradiégétique, une certaine Virginie, précieuse romanesque de province) et son destinataire (un gentilhomme bourgeois fantasque en diable), justifieraient à eux seuls une analyse.

I2. Je reprends les théories de U. Heidmann développées dans Textualité et intertextualité des contes, notamment celle de la (re)configuration générique du conte, celle du «dialogue et [du] dialogisme intertextuel», ainsi que la "conception discursive et dialogique» du conte qui permet "de concevoir l'intertextualité des contes autrement que sur le mode de l'influence et de l'emprunt» (p. 37). 
Kinder-und Hausmärchen, une expression nouvelle. Nous analyserons dans quelle mesure ces contes appartiennent au corpus dont les Grimm disent dans leur préface au premier volume qu'il aurait été utilisé "comme une matière pour construire des récits plus longs, qui ont été enrichis et modifiés arbitrairement ${ }^{13}$ " ("man hat sie fast immer nur als Stoff benutzt, um grössere Erzählungen daraus zu machen, die willkürlich erweitert, verändert [wurden]») et dont les Grimm auraient retrouvé, et recréé, toute leur fraîcheur enfantine et populaire. Cet élan qui a poussé les Grimm à se réclamer explicitement d'un corpus national et à donner à l'Allemagne un recueil qui lui manquait, se radicalise encore trois ans plus tard ${ }^{14}$. Dans leur "Préface à la première édition du tome 2 " paru trois ans plus tard, ils clameront que « tout ce qui a été puisé dans la tradition orale et réuni ici est purement allemand pour sa naissance et sa mise en forme (à l'exception peut-être du Chat botté) ${ }^{15}$ " ("Alles aber, was aus mündlicher Ueberlieferung hier gesammelt worden, ist sowohl nach seiner Entstehung als Ausbildung (vielleicht darin den gestiefelten Kater allein ausgenommen) rein deutsch und nirgends her erborgt $\left.[\ldots]^{16} »\right)$.

Il ne s'agit pas ici de faire une démonstration — inutile — d'influences ou de déni d'influence, mais plutôt d'écouter attentivement le dialogue intertextuel générique ${ }^{17}$ trans-national et trans-séculaire qu'entretiennent ces trois contes.

Avant d'aborder la spécificité poétique du conte, situons-le d'abord dans son contexte péritextuel pour mieux comprendre les préoccupations esthétiques et socioculturelles qui sont celles des Grimm en I8I2. Des trois auteurs de contes entretenant des rapports intertextuels avec Rapunzel — Basile, La Force et Aulnoy — les frères Grimm mentionnent, dans leur préface à la première édition du premier tome de leurs contes, Basile et Aulnoy. Ils situent leurs commentaires sur l'œuvre de Basile dans le contexte plus large du conte italien :

13. Grimm, Contes pour les enfants et la maison, t. I, p. 479.

I4. Dans la "Préface à la première édition du tome I" (I8I2), ils déclaraient déjà : "Nous nous sommes efforcés de saisir ces contes en les laissant aussi purs que possible [...]. Il n'existe encore aucun recueil de ce genre en Allemagne." (Contes pour les enfants et la maison, t. I, p. 479.) ("Wir haben uns bemüht, diese Märchen so rein als möglich war aufzufassen [...]. In diesem Sinne existiert noch keine Sammlung in Deutschland, [...]", Grimm, Kinder- und Hausmärchen, I8I2, p. XVIII-XIX.)

I5. Grimm, «Préface à la première édition du tome 2 (I8I5)», p. 484.

16. Grimm, Kinder- und Hausmärchen, I815, p. XI.

17. Voir la première partie, «Intertextualité et dialogicité des contes», et en particulier, «Le concept de (re)configuration générique», et «Le concept de dialogue intertextuel », par U. Heidmann dans U. Heidmann et J.-M. Adam, Textualité et intertextualité des contes, 20Io, p. 34-40. 
Reicher als alle anderen sind ältere italiänische Sammlungen. Erstlich in den Nächten des Straparola, die manches gute enthalten, dann aber besonders im Pentamerone des Basile, einem in Italien eben so bekannten und beliebten, als in Deutschland seltenen und unbekannten, im neapolitanischen Dialect geschriebenen, und in jeder Hinsicht vortrefflichen Buch. Der Inhalt ist fast ohne Lücke und falschen Zusatz, der Stil überfliessend in guten Reden und Sprüchen ${ }^{18}$.

Les vieux recueils italiens sont plus riches que tous les autres, à commencer par les Nuits de Straparola, qui comportent beaucoup de bonnes choses, mais surtout le Pentamerone de Basile; ce livre aussi connu et apprécié en Italie qu'il est rare et inconnu en Allemagne, est écrit en dialecte napolitain et est excellent à tous les égards. Son contenu ne comporte presqu'aucune lacune ni aucun ajout inapproprié, et le style regorge de belles tournures et de proverbes ${ }^{19}$.

La qualité du recueil de Basile consiste donc dans le fait qu'il ne contient ni retrait ni ajout par rapport à une tradition orale sans doute, mais qui n'est pas mentionnée explicitement. Par contre, qui est, ne fût-ce que vaguement familier avec Lo cunto de li cunti, sera étonné par la déclaration des Grimm selon laquelle il n'y a chez Basile rien d'inapproprié. Si on s'en tient à une putative pureté de transcription de contes oraux, ces contes ne contiennent peut-être pas d'ajout "inapproprié», mais la langue, les métaphores ou encore les discours rapportés contiennent des éléments inappropriés qu'on ne retrouve ni chez Perrault, ni chez les conteuses louis-quatorziennes, et encore moins chez les Grimm. Pour ne prendre qu'un exemple, le contredon du conte Le tre fate (Les Trois fées) qui se manifeste dans la tradition après Basile par le don fait à l'anti-héroïne de produire à chaque parole des crapauds et des vipères, prend chez Basile une forme particulière inconnue après lui. Au lieu de recevoir la malédiction de produire, non pas des pierres précieuses, mais des animaux répugnants et vénéneux, elle reçoit sur le front, non pas comme l'héroïne, une étoile d'or ("na stella d'oro ${ }^{20}$ ") mais... un testicule d'âne ("no testiculo d'aseno $\left.{ }^{2 \mathrm{I}} »\right)$. Non content de ce détail scabreux, le narrateur poursuit en déclarant que cet appendice génital de l'âne qui trône désormais sur le front de l'anti-hérö̈ne serait le résultat d'une «envie que sa mère aurait eue quand elle était grosse» («pareva golio venuto alla mamma quanno era prena $\left.{ }^{22} »\right)$. Lo cunto de li cunti regorge en effet de détails scabreux qui ne sont plus d'actualité ni à l'époque de Perrault et encore moins à celle des frères Grimm.

\footnotetext{
I8. Grimm, ouvr. cité, I8I2, p. XVII-XVIII.

19. Grimm, "Préface à la première édition du tome 2 (I8I5)", p. 478.

20. G. Basile, Lo cunto de li cunti, I999, p. 622.

2I. Ibid.

22. Ibid., p. 624.
} 
L'allusion à la production nationale avec laquelle les frères Grimm entretiennent des rapports intertextuels évidents se fait dans les mêmes termes d'un respect de la simplicité originelle du conte :

Frankreich hat gewiss noch jetzt mehr, als was Charles Perrault mitteilte, der allein sie noch als Kindermärchen behandelte (nicht seine schlechteren Nachahmer, die Aulnoi, Murat); er giebt nur neun, freilich die bekanntesten, die auch zu den schönsten gehören. Sein Verdienst besteht darin, dass er nichts hinzugesetzt und die Sachen an sich, Kleinigkeiten abgerechnet, unverändert gelassen; $[\ldots]^{23}$.

La France a certainement beaucoup plus de contes que ceux transmis par Charles Perrault, qui était le seul à les traiter comme des contes pour enfants (à l'inverse de ses imitateurs moins talentueux, Mme d'Aulnoy, Murat); il n'en donne que neuf, qui sont certes les plus connus et qui font partie des plus beaux. Son mérite consiste à n’y avoir rien ajouté et à avoir laissé les choses telles quelles, excepté quelques détails $[\ldots]^{24}$.

Alors que Perrault, comme Basile, aurait respecté la simplicité du conte oral sans rien y ajouter, Marie-Catherine d'Aulnoy et sa contemporaine Henriette-Julie de Murat auraient «corrompu» le conte originel. «En soi, rien n'est plus difficile, pour la langue française [...] que d'être naïve et directe», déclarent encore les Grimm. Chacun dans leur langue, Basile et Perrault auraient chacun réussi à respecter la simplicité et la naïveté des contes originels alors qu'Aulnoy et sa consœur Murat, n'auraient pas réussi dans cet exercice périlleux de simplicité. D’une autre conteuse, contemporaine aussi de Perrault, Aulnoy et Murat, Charlotte-Rose de Caumont de La Force, ils ne parleront que dans le troisième volume des Kinder- und Hausmärchen, dans une partie consacrée aux «Imitateurs» de Perrault (Nachahmer) ${ }^{25}$.

Nous allons à présent analyser les trois contes du même conte-type 3io, "Persinette", à l'aune de ces déclarations péritextuelles et préfacielles qui encodent la lecture à faire des contes qui suivent et dont nous fêtons le bicentenaire.

\section{Un cas original de «reconfiguration générique»: Petrosinella, Persinette et Rapunzel}

Petrosinella, divertissement premier de la deuxième journée de Lo cunto de li cunti de Giambattista Basile, Persinette, deuxième conte des Contes des

23. Grimm, ouvr. cité, I8I2, p. XVI.

24. Ibid. et Grimm, "Préface à la première édition du tome I (I8I2)», p. 478.

25. Cette partie, considérablement augmentée dans le troisième volume (1822/1856) que l'édition des KHM chez J. Corti a omis de traduire, est donnée en français dans la contribution infra de M. L. Nùñez. 
contes de Charlotte-Rose de Caumont de La Force et Rapunzel, douzième conte des Kinder- und Hausmärchen des Grimm permettent d'approcher l'analyse générique des dialogues intertextuels d'une manière qui n'oppose pas l'approche folklorique du conte-type à une approche littéraire dialogique. Nous ne retenons pas ici les notions de conte oral opposé à l'écrit, ni celle de version originelle ou antérieure, et donc pas celles d'emprunt ou d'influence non plus. Mais nous retiendrons dans notre analyse dialogique générique les transformations formelles effectuées par la conteuse louisquatorzienne et les conteurs allemands dans le contexte des préoccupations poétiques, esthétiques, socioculturelles et morales qui sont les leurs. Cette analyse nous permettra de reformuler un double postulat énoncé jadis par Paul Delarue et Marie-Louise Ténèze selon lesquels : I. « $\mathrm{M}^{\text {lle }}$ de La Force n'a donc pas inventé ce conte comme elle le prétend [...] elle paraît avoir remanié seulement la fin du conte» et 2 . Une déclaration mise sous la caution d'une mise entre parenthèses : "(Cette version [Persinette] semble avoir influencé celle de Grimm en certaines de ses parties ${ }^{26}{ }^{2}$.

J'ai divisé mon analyse des trois contes selon les éléments formels suivants : (I) Le couple parental et l'agresseur, (2) Naissance de l'héroïne et la promesse, (3) La rencontre, (4) Sortie de la tour, exil et pénitence et, finalement, (5) Dénouement et moralité.

\section{Le couple parental et l'agresseur}

Chez les frères Grimm ainsi que chez La Force, le couple parental n'est pas inscrit dans la tradition royale du conte de fées, contrairement à la version d'Aulnoy (un roi et une reine). Chez Basile, il n'y a pas de couple, mais une mère seule qui se voit désignée par un prénom, Pascadozia. Chez La Force, Aulnoy et les Grimm les deux personnages restent, conformément à la tradition du conte, anonymes. Le conte gagne en universalité par la non-désignation spécifique du personnage de la mère. Les Grimm ainsi que La Force ne situent ce conte ni dans la tradition populaire, ni dans la tradition royale.

Mais derrière cette similitude d'éléments qu'on pourrait appeler «Le couple parental» apparaît déjà une reconfiguration par les frères Grimm de la reconfiguration antérieure par La Force :

26. P. Delarue et M.-L. Ténèze, Le Conte populaire français, I997, t. I, p. I8I et I79. C’est moi qui souligne. 
Deux jeunes amants s'étaient mariés ensemble après une longue poursuite de leurs amours; rien n'était égal à leur ardeur, ils vivaient contents et heureux, quand pour combler leur félicité, la jeune épouse se trouva grosse, et ce fut une grande joie dans ce petit ménage ${ }^{27}$.
Es war einmal ein Mann und eine Frau, die wünschten sich schon lange vergeblich ein Kind, endlich machte sich die Frau Hoffnung, der liebe Gott werde ihren Wunsch erfüllen ${ }^{28}$.

Il était une fois un homme et une femme qui désiraient avoir un enfant depuis longtemps déjà, mais en vain. Finalement, la femme eut espoir que le Bon Dieu allait exaucer leur désir ${ }^{29}$.

La Force reconfigure déjà le couple parental d'une triple manière. Elle ajoute un mari absent chez Basile, qui deviendra l'auxiliaire de la transgression à la fois dans Persinette et Rapunzel. Ensuite, elle s'éloigne du sujet populaire et de la spécificité du prénom présents chez Basile. Finalement, elle inscrit le couple parental dans le registre romanesque sentimental : la naissance heureuse se situe dans le cadre d'une "longue poursuite de leurs amours" et d' "ardeurs». Plus d'un siècle plus tard, un tel discours sentimental est devenu anachronique, et il n'a plus sa place dans un conte de fées qui s'adresse aussi à des enfants. Il est reconfiguré chez les Grimm par l'expression d'un désir d'enfant, et sous la protection du «Bon Dieu» ("des lieben Gottes»). On est passé de la Carte de Tendre, de longues amours et d'ardeurs récompensées à un désir d'avoir un enfant et l'espoir d'une bénédiction divine.

La figure de l'agresseur, mais aussi la transgression elle-même, sont radicalement différentes chez Basile et chez les deux autres conteurs. Chez Basile, il s'agit d'une ogresse alors que chez La Force, c'est une fée et chez les Grimm, une magicienne ("Zauberin») 30. L'élément anthropophage associé à l'ogresse a disparu dès les premiers contes de fées littéraires français, il est donc absent chez les Grimm. Ceux-ci n'utilisent pas le mot "fée » mais une périphrase qui définit la fonction de la fée dans l'univers du conte : c'est une "magicienne qui avait un pouvoir immense et que le monde entier craignait ${ }^{31}$ ( ("Zauberin, die grosse Macht hatte und von aller Welt gefürchtet

27. C.-R. de La Force, Les Contes des contes, p. $33 \mathrm{I}$.

28. Grimm, Kinder- und Hausmärchen, I857, p. 87.

29. Grimm, Contes pour les enfants et la maison, p. 80.

30. Pour l'utilisation différenciée de la figure de la fée chez Perrault et des weise Frauen (femmes sages) chez les Grimm, voir l'article de C. François «Fées et weise Frauen : les Faiseuses de dons chez Perrault et les Grimm», 20II.

3I. Grimm, Contes pour les enfants et la maison, p. 80. 
war $\left.{ }^{32} »\right)$. La figure de l'ogresse présente ailleurs dans l'univers féerique, notamment Le Petit Poucet, Finette Cendron et La Belle au bois dormant, n'a pas sa place dans le conte de La Force ou des Grimm où l'enlèvement de l'héroïne a pour but de protéger celle-ci de son propre destin qui reste inexpliqué dans les deux contes.

Chez La Force et les Grimm, l'introduction d'un mari permet un transfert de la transgression de la mère vers le père. Ici encore, La Force a reconfiguré le conte sur le mode romanesque. Après l'aveu par la mère de son irrésistible envie de persil, «le mari soupira et se troubla pour une envie si malaisée à satisfaire; néanmoins comme rien ne paraît difficile en amour...", le mari finit par accéder à la demande extravagante de sa femme. Chez les Grimm, ce discours de soupirs, de trouble, fait place à une considération pratique : "Son mari, qui l'aimait, se dit: "Plutôt que de laisser mourir ta femme, tu vas aller lui chercher des raiponces, quel que soit le prix à payer" ${ }^{33}$.» ("Der Mann, der sie liebhatte, dachte: „Eh du deine Frau sterben lässt, holst du ihr von den Rapunzeln, es mag kosten, was es will“"34.») L'ironie présente en ce seul endroit dans Persinette — «Il fallait que, dans ce temps-là, le persil fût d'un goût excellent" - a disparu de la version des Grimm.

La reconfiguration de la transgression par La Force a un effet direct sur le récit : ce n'est donc pas la mère mais le père qui promet l'enfant à la fée/ magicienne. La mère qui donne son enfant à la fée/magicienne juste après la naissance obéit donc au mari et rien n'est dit sur la difficulté de ce don, contrairement à la version de Basile où la mère refuse d'obtempérer pendant 7 ans, ou encore celle d'Aulnoy où il faut qu'un dragon menace de dévorer tous les sujets du roi pour que l'enfant soit enfin cédé.

\section{Naissance de l'hérö̈ne et la promesse}

Une analyse comparée de la naissance de l'héroïne chez Basile, La Force et les Grimm indique des liens intertextuels entre les contes de La Force et des Grimm, et elle permet d'identifier des reconfigurations significatives.

Chez Basile, l'ogresse n'assiste pas à l'accouchement. Quand elle a 7 ans, Petrosinella commence à fréquenter un maître d'école et elle rencontre en chemin l'ogresse qui charge la fille de rappeler à sa mère sa promesse. Elle le répète si souvent qu’à la fin, la mère, "excédée d'entendre ce refrain, dit à sa fille : "Si tu rencontres encore la vieille, et qu' elle t'interroge sur cette

32. Grimm, ouvr. cité, I857, p. 87.

33. Grimm, Contes pour les enfants et la maison, p. 80.

34. Grimm, ouvr. cité, I857, p. 87. 
maudite promesse, réponds-lui donc : 'En bien! Prends-la”" 35 " ( Si te scuntre co la solita vecchia et te cercarrà sta mardetta prommessa e tu le respunne: - Piglatella! $\left.{ }^{16} »\right)$. L'ogresse saisit la fille "par les cheveux et l'emporta avec elle dans un bois [...] puis elle l'enferma dans une tour qu' elle fit surgir comme par enchantement ${ }^{37}$ " ("e l'orca, afferratala pe li capille, se ne la portaie a no vosco $[\ldots]$ mettennola drinto a no torre che fece nascere ped arte, $\left.[\ldots]^{38} »\right)$.

Le baptême chez La Force et les Grimm indique une double reconfiguration. Tout d'abord, il y a ici une intensification dramatique. La fée/ magicienne, absente chez Basile, est présente lors de l'accouchement, et c'est elle qui donne à l'héroïne son nom. Cette présence et ce don mettent en évidence l'emprise de la fée/magicienne et elle minimise encore la présence parentale. Dès sa naissance, l'héroïne est remise à sa marraine. La Force et les Grimm concentrent donc l'intrigue sur un élément fondateur : le don de l'héroïne en échange du persil ou de la raiponce, et son destin, dès sa naissance, sous l'égide de la fée/magicienne.

Mais cette reconfiguration de la présence de la fée/magicienne commune à La Force et aux Grimm souligne également des différences radicales dont ne peut pas rendre compte l'élément considéré dans le cadre du conte-type : «I. La fillette promise à la fée - D : Elle est invitée au baptême; Di : on donne un nom à l'enfant ${ }^{39}$ ». La fée n'est pas invitée, elle s'invite, et ce n'est pas un anonyme "on» qui lui donne un nom, mais la fée/magicienne. De plus, et la reconfiguration est encore plus significative, les modalités du baptême révèlent une reconfiguration radicale par La Force, et plus tard, une seconde reconfiguration de cette reconfiguration par les Grimm.

Chez les Grimm, la fée qui est apparue aussitôt après l'accouchement «donna à l'enfant le nom de Rapunzel et l'emmena avec elle ${ }^{40}$ " ( gab dem Kinde den Namen 'Rapunzel' und nahm es mit sich for $t^{4 \mathrm{I}}$ ). Chez La Force a lieu tout un cérémonial traduisant une obsession nobiliaire pour la distinction qui se manifeste ici par l'exhibition de signes extérieurs de richesse : la fée reçoit Persinette «dans des langes de toile d'or, et lui a arrosa le visage d'une eau précieuse qu'elle avait dans un vase de cristal, qui la rendit, au moment même, la plus belle créature du monde ${ }^{42} »$. Ce n'est qu'après ces

35. G. Basile, Le Conte des contes, I995, p. I46.

36. G. Basile, Lo cunto de li cunti, I999, p. 286.

37. G. Basile, ouvr. cité, I995, p. I47.

38. G. Basile, ouvr. cité, I999, p. 286.

39. P. Delarue et M.-L. Ténèze, ouvr. cité, p. 178.

40. Grimm, Contes pour les enfants et la maison, p. 8I.

4I. Grimm, ouvr. cité, 1857, p. 87.

42. C.-R. de La Force, ouvr. cité, p. 332. 
«cérémonies de beauté» que la fée emmène l'héroïne. Nous sommes, à la fin du XVII ${ }^{e}$ siècle, dans la société aristocratique louis-quatorzienne, bien loin de «la touffe de l'herbette en question» qu'avait Petrosinella sur la poitrine à sa naissance. Mais ces langes d'or, eaux précieuses et autres cérémonies de beauté qui ont remplacé la prosaïque touffe de persil chez Basile, n’ont plus cours au XIX ${ }^{\mathrm{e}}$ siècle alors que le conte ne s'adresse plus à un lectorat aristocratique adulte imprégné de romans héroïques et de descriptions des cérémonies grandioses de baptêmes féeriques.

Par une même concentration de l'intrigue qui passe sous silence l'éducation de l'héroïne contrairement au conte de Basile où elle n'est enlevée qu'à l'âge de 7 ans, Persinette et Rapunzel sont enfermées dans une tour à l'âge de 12 ans. Les trois tours ont une même spécificité : elles sont hautes, elles n'ont pas de porte mais uniquement une fenêtre très haut placée. Cette spécificité justifie un élément central du conte : la montée dans la tour par les cheveux de l'héroïne.

Mais cette tour est-elle "absolument identique» comme le déclare R. Robert au sujet des contes de Basile et de La Force ${ }^{43}$ ? La décomposition du conte selon ses éléments, "II. La jeune fille dans la tour - A. La fée met la jeune fille dans une tour; $A_{1}$ : qui n'a pas de porte; $A_{2}$ : où elle se fait monter par les longs cheveux de la belle ${ }^{44} »$, invite à une telle conclusion. Mais elle ne permet pas de rendre compte des reconfigurations dialogiques entre les versions. Nous ne savons rien de la tour chez Basile si ce n'est que l'ogresse la « fit surgir comme par enchantement, sans portes, sans escaliers, avec une unique fenêtre, minuscule, où elle montait et descendait en glissant le long des cheveux de Fleur-de-Persil ${ }^{45}$ " "che fece nascere ped arte, senza porte, né scale, sulo co no fenestriello, pe quale pe li capille de Petrosinella, ch'erano luonghe luonghe, saglieva e scenneva, $\left.[\ldots]^{46} »\right)$.

Chez La Force, la tour est inscrite dans le même registre que le baptême : tout, ici, est encore signe de richesse. La tour est "d'argent", elle contient «de grands et beaux appartements aussi éclairés que si la lumière du soleil y fût entrée, et qui recevaient le jour par le feu des escarboucles dont toutes ces chambres brillaient. Tout ce qui était nécessaire à la vie s'y trouvait splendidement; toutes les raretés étaient ramassées dans ce lieu ${ }^{47}$ ». Tout, ici, est superlatif, des "plus beaux bijoux» aux "garde-robes [...] magnifiques»,

43. R. Robert, art. cité, p. 339.

44. P. Delarue et M.-L. Ténèze, ouvr. cité, p. 178.

45. G. Basile, ouvr. cité, I995, p. I47.

46. G. Basile, ouvr. cité, I999, p. 286-288.

47. C.-R. de La Force, ouvr. cité, p. 332. 
aux «mets les plus délicieux ${ }^{48}$ ». L'éducation même de l'hérö̈ne tient du stéréotype de l'époque : l'héroïne "ne s'ennuyait pas dans sa solitude; elle lisait, elle peignait, elle jouait des instruments et s'amusait à toutes ces choses qu'une fille qui a été parfaitement élevée n’ignore point ${ }^{49}$ ». Les Grimm ne reprendront ni le décor superlatif, ni l'éducation romanesque digne d'un roman précieux ou héroïque. Non seulement les conventions esthétiques et romanesques ne sont, à leur époque, plus les mêmes, mais les préoccupations socioculturelles d'une aristocrate de la fin du XVII ${ }^{\mathrm{e}}$ siècle ne sont plus les mêmes au début du XIX ${ }^{\mathrm{e}}$ siècle où l'éducation des enfants est prise plus au sérieux et où l'éducation "parfaite» d'une héroïne n'est forcément plus la même quand elle s'adresse à toutes les petites filles.

\section{La rencontre}

La scène de la rencontre avec le prince indique un triple décalage entre les Grimm et le conteur napolitain, et un triple parallèle intertextuel avec la version de La Force. Ces éléments sont : le chant incantatoire, la rencontre initiale et la manière dont cette rencontre se réalise.

Chez Basile, seule l'apparence de la chevelure attire l'attention du fils du prince. Petrosinella passe sa tête par la fenêtre de la tour et répand ses tresses au soleil :

[...] vedenno doie bannere d'oro che chiammavano l'arme ad assentarese a lo rollo d'Ammore e miranno drinto a chelle onne preziose na facce de Serena che 'ncantava li core, se 'ncrapecciaie fora de mesura de tanta bellezze; [... $]^{\text {so }}$.

[...] avisant ces deux drapeaux dorés qui incitaient les âmes à s'enrôler dans les armées d'Amour, et, au milieu de ces ondes précieuses, un visage de Sirène qui ensorcelait les cours, s'enticha follement de cet amoncellement de beautés; $[\ldots]^{\text {sI }}$.

Chez La Force et les Grimm, c'est la voix de l'héroüne qui attire le prince. Chez La Force, cette reconfiguration découle de la mention qui précède de la "voix divine» de Persinette, jeune fille dont l'éducation parfaite selon les goûts du temps est détaillée. Les Grimm, qui n’ont rien dit de cette éducation, ont cependant conservé cette reconfiguration.

La scène de la princesse enfermée dans la tour peut mettre en scène deux figures d'héroïnes. Une première qui n'a pas froid aux yeux, observe le prince sans gêne ni peur, et elle peut aller jusqu'à initier un dialogue, voire à inviter

48. Ibid., p. 332-333.

49. Ibid., p. 333.

50. G. Basile, ouvr. cité, I999, p. 288.

5I. G. Basile, ouvr. cité, 1995, p. I47. 
le prince à la rejoindre. C'est le cas de la Petrosinella de Basile. Ce modèle est toujours pertinent pendant la première vague du conte de fées littéraire, puisque c'est le modèle que suit l'héroïne d'Aulnoy dans Chatte Blanche.

La princesse de La Force et celle des Grimm est plus retenue. Chez La Force, cependant, le registre qui décrit l'observation de l'héroïne s'inscrit une fois encore dans le romanesque et le sentimental : "Persinette, de son côté, perdit la parole quand elle vit un homme si charmant, elle le considéra longtemps tout étonnée, mais tout à coup, elle se retira de la fenêtre, croyant que ce fût quelque monstre, se souvenant d'avoir ouï dire qu'il y en avait qui tuaient par les yeux, et elle avait trouvé les regards de celui-ci très dangereux ${ }^{52}$.» Dans le contexte de galanterie qui est le sien, La Force a reconfiguré les échanges entre l'héroöne et le prince : plus question d'un "amoncellement de beautés» et encore moins d'un "grimoire de soupirs\$" comme c'est le cas chez Basile. Les prosaïques «baisers volants», les «clignements d'yeux», les "salamalecs " présents ici ont été ici reconfigurés en un stéréotype de l'amour qui tue et de l'amour qui enchaîne par le regard.

Les Grimm feront de leur héroïne un personnage encore plus réservé qui, non pas comme chez Basile ou Aulnoy, invite le prince à monter dans la tour, ni comme l'héroïne de La Force, observe le Prince mais se réfugie dans la tour pour éviter de "mourir d'amour». Rapunzel ne voit pas le prince, ce qui rend la tromperie de celui-ci et la surprise de l'héroïne lors de l'arrivée du prince en haut de la tour encore plus surprenantes pour l'héroïne, et garantit sa naïveté et son innocence.

Chez La Force et les Grimm, le prince contrefait la voix de la fée/ magicienne et il prononce les paroles incantatoires : ici, «Percinette, descendez vos cheveux que je monte» et là, "Raiponce, Raiponce, / déroule tes cheveux pour que je monte» ("Rapunzel, Rapunzel, lass dein Haar herunter $\left.{ }^{54} »\right)$. La Force opère ici encore une reconfiguration du thème de la rencontre selon le goût du jour plus enclin à la pudeur. Chez Basile, le prince «s'engouffra dans la chambre pour déguster un peu de persil à la sauce Amour"s" ("schiaffatose pe lo finestrello dintro la cammara, si fece no pasto de chillo petrosino de la sauza d'Ammore $\left.[\ldots]^{56} »\right)$. Le couple héroïque prend tant de plaisir à ces plats dévorés «à la sauce Amour» qu'une "commère de l’ogresse» qui voulait «fouiner dans la merde des autres» prévient celle-ci

52. C.-R. de La Force, ouvr. cité, p. 333.

53. G. Basile, ouvr. cité, I995, p. I47.

54. Grimm, ouvr. cité, I857, p. 89.

55. G. Basile, ouvr. cité, 1995, p. I47.

56. G. Basile, ouvr. cité, 1999, p. 288. 
$\mathrm{du}$ «trafic et remue-ménage» qu' elle entend tous les soirs ${ }^{57}$ ("na commare dell'orca [...] voze mettere lo musso a la merda [...] vedeva lo moschito e lo trafeco che se faceva $\left.[\ldots]^{58} »\right)$.

Chez La Force, le langage est à la fois sentimental et plus pudique. Arrivé en haut de la tour, le prince "pensa en tomber en bas, quand il remarqua de si près cette prodigieuse beauté [...] il lui embrassa les genoux avec une ardeur qui pouvait la persuader ${ }^{59}$ ». D'abord effrayée, Persinette se trouble mais se laisse finalement persuader et elle accepte la proposition du Prince de l'épouser dans l'heure: "Elle y consentit sans savoir presque ce qu'elle faisait, elle acheva de même toute la cérémonie ${ }^{60}$." Il y a bien consommation du mariage chez La Force, ce qui entraînera la grossesse, l'exil et la pénitence de l'héroïne. Elle est décrite dans des termes indirects qui font référence à une ignorance plutôt qu'à une consommation enthousiaste, quotidienne, répétée, et bruyante.

Le mariage conclu prend une forme plus pudique encore chez les frères Grimm qui en ont adouci les connotations sexuelles au fil des versions, se rapprochant finalement, dans la dernière version, de la pudeur de l'héroïne de La Force. La première édition est plus explicite : Rapunzel et le prince "vécurent donc dans la joie et le plaisir pendant un certain temps, et la magicienne ne se rendait compte de rien, jusqu'à ce qu'un jour, Rapunzel lui parle en ces termes : "Dites-moi donc, Marraine, mes vêtements sont devenus si étroits et ne me vont plus" "6" ("So lebten sie lustig und in Freuden eine geraume Zeit, und die Fee kam nicht dahinter, bis eines Tages das Rapunzel anfing und zu ihr sagte: "Sag' mir doch Frau Gothel, meine Kleiderchen werden mir so eng und wollen nicht mehr passen "62"). La grossesse, explicitement mentionnée dans cette première version et qui est présente aussi chez La Force, a disparu dans la dernière version de I857. Ici, Rapunzel, d'abord effrayée mais séduite par les propos du prince, pose un geste presque enfantin : "voyant qu'il était jeune et beau, elle acquiesça et mit sa main dans la sienne ${ }^{63}$ " ("sie sah, dass er jung und schön war, [...] und legte ihre Hand in seine $\left.e^{64} »\right)$.

57. G. Basile, ouvr. cité, I995, p. I48.

58. G. Basile, ouvr. cité, 1999, p. 288.

59. C.-R. de La Force, ouvr. cité, p. 335.

6o. Ibid.

6I. Grimm, Contes pour les enfants et la maison, p. 84 .

62. Grimm, ouvr. cité, I8I2, p. 4I.

63. Grimm, Contes pour les enfants et la maison, p. 82.

64. Grimm, ouvr. cité, I857, p. 89. 


\section{Sortie de la tour, exil de l'héroïne et pénitence du héros}

Après la rencontre dans la tour du prince et de l'héroïne, les contes de La Force et des Grimm se séparent radicalement des versions attestées du conte-type. Une analyse du conte sur le modèle folklorique déclare que «la conteuse paraît avoir remanié seulement la fin du conte ${ }^{6{ }^{5}} \%$. Mais c'est ne pas tenir compte de la reconfiguration, non plus de motifs ou de formulations sur l'un ou l'autre registre plus approprié aux préoccupations esthétiques ou socioculturelles qui sont celles de La Force et de ses contemporains, mais bien une reconfiguration de la structure même du conte qui a des conséquences essentielles pour la signification non seulement poétique mais également idéologique de cette reconfiguration. Celle-ci concerne la fuite de la tour, l'exil de l'héroïne, la pénitence du couple héroïque et le dénouement du conte.

Contrairement à la version de Basile et aux versions inspirées de contes oraux ou folkloriques, il n'y a pas chez La Force et les Grimm de fuite du couple héroïque. Cette fuite rocambolesque chez Basile et qui a des connotations intertextuelles avec d'autres contes (l'ogresse porte une peau d'âne et se fait dévorer par un loup) a pour conséquence de mettre le couple à l'abri de la fureur de l'ogresse trompée, et de laisser ainsi et la consommation du mariage et la fuite du couple sans punition aucune. Contrairement aux versions de La Force et des Grimm, il n'y a dans le conte de Basile aucune punition de l'héroïne, aucune rétribution pour son comportement. Petrosinella réussit à s'enfuir de la tour :

E li 'nammorate scenno de 'impaccio se ne iettero chiano chiano a lo regno de lo prencepe, dove, co bona lecenzia de lo patre, se la pigliaie pe mogliere $[\ldots]^{66}$.

[...] les amoureux, enfin sortis d'ennuis, s'en allèrent piane-piane vers le royaume du jeune homme, où, avec la permission de son noble père, le prince prit Fleur-de-Persil pour épouse $[\ldots]^{67}$.

Pas d'enfants ici, contrairement aux versions de La Force et des Grimm, ni aucune épreuve, ni pour le prince, ni pour l'héroïne grâce au don des trois glands. Ironiquement, le don qui permet au couple héroïque d'échapper au courroux de l'ogresse est fait par l'agresseur qui en révèle la présence et la localisation, permettant au prince de s'en emparer. Ce don par l'agresseur

65. P. Delarue et M.-L. Ténèze, ouvr. cité, p. I8I.

66. Basile, ouvr. cité, 1999, p. 292.

67. Basile, ouvr. cité, I995, p. I49. 
rature implicitement la punition qui n'est donc jamais infligée, contrairement aux versions de La Force et des Grimm.

La transgression par l'héroïne de l'interdit, mais aussi le mariage consenti et sa consommation ne peuvent plus chez La Force ni chez les Grimm rester impunis. Chez La Force, la fée remarquant l'état de Persinette, se doute de la faute commise avec le prince et «point touchée de tout l'amour dont Persinette lui racontait des traits si touchants », elle la fait descendre de la tour et l'emmène dans une contrée lointaine et «très solitaire, mais assez agréable ${ }^{68} »$.

Chez les Grimm, ce n'est pas l'état de l'héroïne qui la trahit, mais l'héroïne elle-même qui avoue naïvement qu'il lui est plus facile de hisser le prince que la magicienne. Cette reconfiguration a un double effet. Tout d'abord, la naïveté enfantine de l'héroïne lorsqu'elle consent à épouser le prince — «elle mit sa main dans la sienne» ("und legte ihre Hand in seine») - est confirmée dans cet aveu à la magicienne : "J'ai bien plus de mal à vous hisser jusqu'ici que le fils du roi qui, lui, est en haut en un clin d'œil ${ }^{69}$ " ( Sie wird mir viel schwerer heraufzuziehen als der junge Königsohn, der ist in einem Augenblick bei mir $\left.{ }^{70} »\right)$. De plus, cet aveu court-circuite l'allusion à la grossesse qui se retrouve ainsi rejetée en fin de conte lorsque nous apprenons que Rapunzel "vivait misérablement avec les jumeaux, un garçon et une fille, qu'elle avait mis au monde ${ }^{71}$ " ("wo Rapunzel mit den Zwillingen, die sie geboren hatte, einem Knaben und einem Mädchen, kümmerlich lebte $\left.{ }^{72} »\right)$. L'allusion à la grossesse de l'héroïne dans un conte mondain pour adultes a pour effet de faire bifurquer radicalement le conte de toutes les autres versions antérieures connues. À partir du troisième élément, "La fuite et ses conséquences", la reconfiguration de La Force se différencie en effet de toute autre version antérieure écrite ou orale. Mais cette allusion qui pouvait choquer la morale louis-quatorzienne au point de justifier une double (triple chez La Force) pénitence, n’a plus sa place dans un conte destiné aussi aux enfants.

Quand l'héroïne a été emmenée dans l'endroit de son exil, la fée/magicienne revient à la tour d'une manière identique chez la conteuse française et les conteurs allemands. Elle utilise le même stratagème pour tromper le prince que celui-ci avait utilisé pour monter dans la tour :

68. C.-R. de La Force, ouvr. cité, p. 335-336.

69. Grimm, Contes pour les enfants et la maison, p. 83.

70. Grimm, ouvr. cité, 1857 , p. 90.

71. Grimm, Contes pour les enfants et la maison, p. 83.

72. Grimm, ouvr. cité, I857, p. 90-9I. 
Elle remonta à la tour, et se mettant à chanter du ton dont chantait Persinette, le prince, trompé par cette voix, et qui revenait pour la voir, lui redemanda ses cheveux, pour monter comme il l'avait accoutumée; la perfide fée les avait exprès coupés à la belle Persinette, et les lui tendant, le prince parut à la fenêtre, où il eut bien moins d'étonnement que de douleur de ne pas trouver sa maitresse ${ }^{73}$. (LF 336)

Chez les Grimm, «le soir où elle avait répudié Rapunzel, la magicienne attacha au crochet de la fenêtre, en haut de la tour, les tresses qu'elle avait coupées. Quand le fils du roi vint et appela : «Raiponce, Raiponce / déroule tes cheveux pour que je monte ${ }^{74}$ ( (Rapunzel, Rapunzel, lass dein Haar herunter»), elle fit dégringoler les cheveux. Le fils du roi monta mais ce n'est pas sa Raiponce bien-aimée qu'il trouva en haut, c'était la magicienne qui le regardait d'un air méchant et fielleux.

Derrière une scène apparemment similaire apparaît une reconfiguration. Chez les Grimm, on s'éloigne une fois encore du discours galant qui fait de la fée une "perfide», et crée chez le prince "moins d'étonnement que de douleur» de ne pas trouver sa bien-aimée. Par contre apparaissent des adjectifs moins galants mais plus pédagogiques et plus évocateurs pour des enfants tels que "méchant et fielleux".

Chez La Force et chez les Grimm, le prince se jette du haut de la tour, alors qu'il s'est enfui chez Basile, et qu’il est dévoré chez Aulnoy. Chez La Force, le discours galant prédomine encore : le prince, "plus agité des fureurs de la douleur que contraint par la puissance de l'art de la fée, se précipita du haut de la tour en bas. Il devait mille fois se briser tout le corps, il tomba sans se faire autre mal que de perdre la vue ${ }^{75}{ }^{\prime}$. Chez les Grimm, la scène est moins galante et inscrite dans un registre de causalité : pas de personnage «agité des fureurs de la douleur» mais tout simplement «Fou de douleur» ("Er geriet ausser sich vor Schmerz»). Concrète, la perte de la vue n'est pas décrite comme une variation d'un métaphorique «se rompre le cou", mais bien le résultat du fait que des épines qui se trouvaient au bas de la tour ont crevé les yeux du prince.

La pénitence du héros est absente chez Basile, grâce à la fuite du couple héroïque aidée par le don de l'ogresse. Elle l'est aussi chez Aulnoy pour le prince dévoré par un dragon. Cette pénitence du prince téméraire est peu propice à l'expression d'élans galants chez La Force, et elle n'est pas reconfigurée chez les Grimm, si ce n’est que, ici, «il se nourrissait des herbes

73. C.-R. de La Force, ouvr. cité, p. 336.

74. Grimm, Contes pour les enfants et la maison, p. 83.

75. C.-R. de La Force, ouvr. cité, p. 336. 
et des racines ${ }^{76}$ » et que là, il ne se nourrissait " que de racines et de baies ${ }^{77}$ » ("ass nichts als Wurzeln und Beeren $\left.{ }^{78} »\right)$. Qu'il s'agisse d'herbes ou de baies, la pénitence reste la même, au mot près. Il n'en va pas de même de l'exil de l'héroïne. Chez La Force, la pénitence de l'héroïne est relative et exprimée dans des termes, une fois encore, romanesques. Convention romanesque aidant, on n'est pas loin du Forez de l'Astrée : «Il y avait des prés, des bois, un ruisseau d'eau douce, une petite hutte faite de feuillages toujours verts. » Convention sociale aristocratique obligeant aussi, on se soucie peu de la nourriture de survie : à côté d'un lit de jonc marin, on trouve ainsi «une corbeille dans laquelle il y avait de certains biscuits qui étaient assez bons, et qui ne finissaient point ${ }^{79}$ ». La pénitence de Rapunzel est reconfigurée dans le contexte de préoccupations de survie bien réelles : elle a lieu "dans une contrée déserte où celle-ci dut vivre dans la détresse et la misère ${ }^{80}$ » ( in die Wüstenei, wo Rapunzel [...] kümmerlich lebte $\left.{ }^{8 \mathrm{I}}\right)$ ).

\section{Dénouement et moralité}

La scène des retrouvailles est occasionnée chez La Force et les Grimm par la voix de l'héroïne que reconnaît le prince, logique narrative oblige puisqu'il est aveugle. Cette réunion qui n'a lieu ni chez Basile (puisque le couple n'est jamais séparé) ni chez Aulnoy (puisque le prince est dévoré par un dragon) semble bien être une invention de La Force. Les configurations - et je parle ici de configurations puisqu'il s'agit, faute de preuve du contraire, d'une invention de La Force que reconfigureront les Grimm - témoignent encore des préoccupations esthétiques des époques respectives où les deux contes ont été écrits.

Cette scène a des connotations sentimentales évidentes chez la conteuse louis-quatorzienne. Quand elle reconnaît le prince, Persinette «fit un cri perçant et s'élançant auprès de lui, son saisissement fut si sensible que, par un effet bien naturel, elle versa un torrent de larmes ${ }^{82}$ ». Les Grimm qui n'écrivent plus pour des aristocrates amateurs de romans héroïques, s'en tiennent à un factuel : "Raiponce le reconnut et se jeta à son cou en pleu-

76. Ibid., p. 337.

77. Grimm, Contes pour les enfants et la maison, p. 83.

78. Grimm, ouvr. cité, 1857 , p. 90.

79. C.-R. de La Force, ouvr. cité, p. 336.

80. Grimm, Contes pour les enfants et la maison, p. 83.

81. Grimm, ouvr. cité, 1857, p. 90.

82. C.-R. de La Force, ouvr. cité, p. 337. 
rant $^{83}$ " («erkannte ihn Rapunzel und fiel ihm um den Hals und weinte ${ }^{84} »$ ). Même les larmes thérapeutiques de l'héroïne qui, dans les deux contes, guérissent le prince de la cécité, connaissent une reconfiguration évidente : chez les Grimm, «deux de ses larmes mouillèrent les yeux du fils du roi, et ils recouvrèrent la vue, de sorte qu'il vit aussi bien qu'avant ${ }^{85}$ » («Zwei von den Tränen aber benetzten seine Augen, da wurden sie wieder klar, und er konnte damit sehen wie sonst $\left.{ }^{86} »\right)$. Nous sommes loin des larmes de Persinette qui coulent «à torrents» et qui ne sont autres, bien entendu, que des «larmes précieuses». Les retrouvailles sentimentales, le registre émotionnel des larmes versées à profusion ont été reconfigurés en une rencontre finale décrite sur un registre factuel : "Il emmena Raiponce dans son royaume où on le reçut dans la joie, et ils vécurent encore longtemps, dans le bonheur et la gaîté ${ }^{87}$ ». ("Er führte sie in sein Reich, wo er mit Freude empfangen ward, und sie lebten noch lange glücklich und vergnügt $\left.{ }^{88} "\right)$.

C'est ici que les Grimm ont reconfiguré le plus radicalement le conte de La Force. La seconde pénitence imposée par la fée dans le conte de La Force est complètement raturée de la version des Grimm. Cette nouvelle pénitence est inscrite, une fois encore, dans un registre romanesque sentimental : au «spectacle bien touchant» des retrouvailles succède un tableau sentimental d'un couple parfait, un "beau prince», "cette charmante princesse et ces aimables enfants » réduits à une cruelle famine. Les termes qui décrivent cette scène sont empruntés au registre pathétique et conduisent à la rédemption finale. Les pauvres enfants sont ainsi réduits à « une défaillance qui les mettait à deux doigts de la mort»: "Qui n'aurait pas été touché de voir ainsi mourante cette déplorable famille ${ }^{89}$ ?» Qui en effet? La réponse est évidente : personne, pas même la fée. Il faut cette mise en scène pour qu'ait lieu le pardon final appelé ici " un miracle favorable», et pour que soit tirée la morale, explicite chez La Force, et absente chez les Grimm :

Tendres époux, apprenez par ceux-ci

Qu'il est avantageux d'être toujours fidèles;

Les peines, les travaux, le plus cuisant souci,

Tout enfin se trouve adouci

Quand les ardeurs sont mutuelles;

83. Grimm, Contes pour les enfants et la maison, p. 83.

84. Grimm, ouvr. cité, 1857, p. 91.

85. Grimm, Contes pour les enfants et la maison, p. 83.

86. Grimm, ouvr. cité, I857, p. 90.

87. Grimm, Contes pour les enfants et la maison, p. 84.

88. Grimm, ouvr. cité, I857, p. 9I.

89. C.-R. de La Force, ouvr. cité, p. 338. 
On brave la fortune, on surmonte le sort,

Tant que deux époux sont d'accord.

La moralité de La Force est inscrite dans le registre des "ardeurs» sentimentales et précieuses dont avait déjà fait preuve le couple parental ("rien n'égalait à leur ardeur»), mais aussi le prince lors de la rencontre avec Persinette ( il lui embrassa les genoux avec une ardeur qui pouvait la persuader $")^{9 \circ}$. De telles considérations galantes et précieuses sont bien éloignées des préoccupations esthétiques et poétiques, mais aussi du contexte idéologique et socioculturel des frères Grimm qui prétendaient avoir puisé leurs sources parmi le peuple, et s'adresser au peuple à qui ils voulaient rendre "tout ce qui s'apparente aux légendes populaires", des contes par et pour le peuple.

\section{Conclusion}

L'analyse du conte selon la méthode du conte-type ne permet pas de rendre compte des reconfigurations génériques spécifiques et du dialogue intertextuel qu'entretiennent les contes de Basile, de La Force et des Grimm. La signification de contes et leurs dialogues intertextuels ne dépendent en effet pas uniquement de la succession d'événements ni des actants agissant, mais également de l'épaisseur textuelle spécifique de chaque conte qui témoigne du contexte esthétique et socioculturel du conteur ou de la conteuse qui lui donne une ou plusieurs forme(s).

Dans leur préface de la première édition du tome I, les Grimm qualifraient le recueil de Basile d' «excellent à tous les égards» dans la mesure où «il ne comporte presqu'aucune lacune ni aucun ajout inapproprié ${ }^{9 r}{ }^{\prime}$. Dans la même préface, les Grimm reconnaissaient aussi la simplicité de Perrault, "à l'inverse de ses imitateurs, mois talentueux, Mme d'Aulnoy, Murat». Le mérite de Perrault consisterait à "n'y avoir rien ajouté et à avoir laissé les choses telles quelles ». Or, rien, selon les Grimm, ne serait plus difficile, pour la langue française, qui est aussi celle de Charlotte-Rose de Caumont de La Force «que d'être naïve et directe, c'est-à-dire de prétendre raconter des contes pour enfants".

Le procès intenté aux «imitatrices» est injuste pour elles : elles n'écrivaient pas pour des enfants mais pour leurs contemporains et contemporaines

90. Je remercie J.-P. Sermain de m'avoir fait remarquer la répétition de cette thématique des «ardeurs».

91. Grimm, "Préface à la première édition du tome 2 (I8I5)», p. 478. 
mondains ou aristocratiques. Non seulement, elles ne racontaient pas des Kindermärchen des "contes pour enfants", mais la tradition littéraire n'était pas non plus au "direct» ni au "naïf». La tradition esthétique était au romanesque sentimental descendu en droite ligne de la tradition héroïque et des revendications de raffinement héritées des Précieuses.

Certes, les frères Grimm ont bien reconfiguré le conte de La Force pour lui donner la forme universelle qu'il a. Qui, aujourd'hui, ne connaît par Rapunzel? Et, inversement, qui connaît encore Persinette?

Certes, il y a chez la conteuse louis-quatorzienne des signes évidents qui trahissent des préoccupations poétiques et idéologiques de l'époque et qui paraissent aujourd'hui historiquement datés. Aux ardeurs des parents succèdent celles du prince et finalement, dans la moralité, celles du couple héroïque. On croit que le regard d'un amant peut tuer, on "pense mourir de douleur" en apercevant une beauté, on perd fort vite la parole, on se jette aisément aux genoux de la bien-aimée qu'on embrasse, on ne pleure pas mais on verse un «torrent de larmes» qui, «Ô, merveille!», sont des larmes... précieuses. Pour le contenu, tout est, ici, infiniment supérieur et signe extérieur de richesse. Vingt ans après la construction de Versailles et en plein soleil déclinant d'un roi qui s'est autoproclamé «Le Roi Soleil», ou "Louis le Grand", sera-t-on surpris que tout, ici, soit plus grand qu'ailleurs, et que tout y brille de mille feux? On s'étonne que la tour dans laquelle est enfermée Persinette ne soit que d'argent, mais elle rappelle la pompe architecturale des monuments érigés dans le royaume pour signifier sa grandeur, y compris ses «appartements aussi éclairés que si la lumière du soleil y fût entrée ${ }^{92} »$. Tout, dans cet univers merveilleux, témoigne d'une superlative ostentation de richesses : on ne voit que langes de toile d'or et eaux précieuses qui garantissent une parfaite beauté à celle qui les reçoit, on ne mange que les mets les plus délicats, on ne chante que merveilleusement, et la voix de l'héroïne ne peut être que divine.

N'oublions pas pour autant que La Force, contemporaine de Perrault, d'Aulnoy et de Murat, conteuse de la première génération qui publiait des contes avant même que ne naisse l'expression générique consacrée aujourd'hui mais qui ne naîtra que l'année suivant la publication de Persinette, avait, elle aussi, déjà reconfiguré le conte de Basile ${ }^{93}$. Si La Force a inscrit sa Persinette dans les préoccupations poétiques et idéologiques qui étaient celles de

92. C.-R. de La Force, ouvr. cité, p. 332.

93. M.-C. d'Aulnoy a publié en I697, l'année de la publication de Persinette, ses Contes des Fées, mais ce ne sera que l'année suivante qu'H.-J. de Castelnau, comtesse de Murat, publiera ses Contes de fées. 
son époque, elle a cependant aussi reconfiguré le conte napolitain d'une manière originale que n'avait connue aucune autre version orale ou écrite avant elle, et qui pourra ensuite, à son tour, être reconfigurée par les frères Grimm plus d'un siècle plus tard.

Dire que «cette version semble avoir influencé celle de Grimm en certaines de ses parties» ne reflète pas la reconfiguration profonde, par La Force, du conte de Petrosinella. La Force a en effet reconfiguré le conte à partir de ses éléments «II. D : La présence est dénoncée par le perroquet", c'est-à-dire dès le milieu même du conte puisque le dernier élément du conte-type est le III. D : «La fée rend figure humaine et beauté à sa filleule».

Basile met en scène une héroïne enfermée dans une tour qui se laisse séduire par un fils de roi à qui elle envoie des baisers volants, des clignements d'yeux et de douces paroles en échange de ses "offres», propositions honnêtes et sans doute moins honnêtes, une héroïne qui hisse son prince en haut de sa tour pour que celui-ci puisse déguster « un peu de persil à la sauce Amour ", et cela tant de fois, avec tant d'ardeur, avec tant d'enthousiasme et de «remue-ménage» qu'une mégère voisine en est alertée. Chez Basile, l'héroïne consomme impunément son mariage et ne subit aucune punition ni pénitence. Elle réussit au contraire à fuir avec son prince pour jouir, sans autre forme de procès, d'une félicité matrimoniale digne d'un conte de fées...

Certes, la reconfiguration de La Force a, dans sa double pénitence et sa moralité précieuse explicite, une dimension romanesque sentimentale teintée de roman héroïque. Mais le couple subit une pénitence : l'héroïne est exilée tandis que le héros, aveugle, est condamné à errer avant de recouvrer la vue, et de retrouver l'héroïne et leurs enfants. Seule cette pénitence peut garantir le conte d'un quelconque sceau de moralité. Les conteurs allemands se sont contentés d'une seule pénitence, et leur conte ne moralise pas explicitement et d'une manière galante sur les ardeurs partagées comme l'avait fait La Force. Cette double reconfiguration rend le conte de I8I2 beaucoup plus contemporain et universel que celui de I697. Ceci n'enlève cependant rien au fait que La Force avait déjà reconfiguré le conte dans son intrigue, et donc dans ses implications morales, et que les Grimm pourraient alors à leur tour en reconfigurer à la fois l'esthétique galante et l'univers aristocratique, tout en en conservant l'intrigue.

Aujourd'hui, alors que règne l'ère de la construction de l'identité européenne dans laquelle s'inscrivent les appartenances nationales, plutôt que d'argumenter sur l'originalité, ou pas, des frères Grimm, sur leur imitation, ou pas, imitation consciente, ou pas, d'un conte aristocratique de l'époque de Louis XIV, nous préférons souligner les reconfigurations de contes, leurs 
dialogues intertextuels qui obéissent aux préoccupations poétiques, idéologiques et socioculturelles qui furent celles de chaque conteur et de chaque conteuse. Nous préférons reconnaitre la contribution originale et visionnaire des frères Grimm qui ont donné, dans leur Rapunzel, un véritable conte franco-allemand, nous livrant un conte non pas "purement allemand pour sa naissance et sa mise en forme" ("rein deutsch und nirgends her erborgt [...]", mais un conte "purement européen», et cela, plus d'un siècle et demi avant la naissance même de l'Europe. Car si les frères Grimm en ont reconfiguré l'expression, c'est qu'une aristocrate française en avait, avant eux, plus d'un siècle plus tôt, reconfiguré la forme.

\section{Ouvrages cités}

\section{Contes}

Aulnoy Marie-Catherine de (Marie Catherine Le Jumel de Barneville), Contes nouveaux ou les Fées à la mode, édition critique établie par Nadine Jasmin, Paris, Honoré Champion, coll. "Champion Classiques», 2008.

Basile Giambattista, Lo cunto de li cunti, traduction et édition de Michele Rak, Milan, Garzanti, I999 [1634-1636].

-, Fleur-de-Persil, dans Le Conte des contes, traduction du napolitain de Françoise Decroisette, Strasbourg, Circé, 1995.

Grimm Jacob et Wilhelm, Raiponce, dans Contes pour les enfants et la maison, édités et traduits par Natacha Rimasson-Fertin, Paris, José Corti, 2009, t. I.

-, Kinder- und Hausmärchen, Ausgabe letzter Hand, édités par Heinz Rölleke, Stuttgart, Reclam, 200I [I856/I857], 3 vol.

—, Kinder- und Hausmärchen. Gesammelt durch die Brüder Grimm, [1812 \& I8I5], réimpression augmentée de la première édition de I8I2 et I8I5 d'après la copie personnelle du Musée des Frères Grimm de Kassel, avec toutes les corrections manuscrites et les modifications des frères Grimm, [...] par Heinz Rölleke, Göttingen, Vandenhoeck \& Ruprecht, I986, 2 vol.

La Force Charlotte-Rose de (Charlotte-Rose de Caumont de La Force), Persinette, dans Contes de Mademoiselle Lhéritier, Mademoiselle Bernard, Mademoiselle de La Force, Madame Durand, Madame d'Auneuil, édition critique établie par Raymonde Robert, Paris, Honoré Champion, coll. «Bibliothèque des Génies et des Fées», 2005, t. 2. 


\section{Ouvrages critiques}

Anderson Benedict, Imagined Communities, Londres, Verso, I983; L'imaginaire national, traduction par Pierre-Emmanuel Dauzat, Paris, La Découverte, 1996.

Delarue Paul et Ténèze Marie-Louise, Le Conte populaire français, Catalogue raisonné des versions de France, t. I, Paris, Maisonneuve et Larose, 1997.

Escola Marc, Marc Escola commente les Contes de Charles Perrault, Paris, Folio, coll. «Foliothèque», 2005.

FrançoIs Cyrille, «Fées et weise Frauen: les Faiseuses de dons chez Perrault et les Grimm, du merveilleux rationalisé au merveilleux naturalisé», dans Martine Hennard Dutheil de la Rochère et Véronique Dasen (éds), Des fata aux fées : Regards croisés de l'Antiquité à nos jours, Lausanne, Revue de la Faculté des lettres de l'Université de Lausanne, Études de lettres, $\mathrm{n}^{\circ} 289$, 20II, p. 259-278.

Heidmann Ute et Adam Jean-Michel, Textualité et intertextualité des contes: Perrault, Apulée, La Fontaine, Lhéritier..., Paris, Classiques Garnier, coll. "Lire le XVII ${ }^{\mathrm{e}}$ siècle», 2010.

Heidmann Ute, «Expérimention générique et dialogisme intertextuel : Perrault, La Fontaine, Apulée, Straparola, Basile, dans Féeries, $n^{\circ} 8$, «Le merveilleux français à travers les siècles, les langues, les continents", dossier coordonné par Jean Mainil, 20II, p. 52-62.

Herman Jan, Kozul Mladen et Kremer Nathalie, Le Roman véritable: Stratégies préfacielles au XVIII siècle, Oxford, Voltaire Foundation, SVEC, 2008.

Le Noble Eustache, "Au lecteur», Ildegerte, Reyne de Norvège, ou l'Amour magnanime, première nouvelle historique, par M. D. *, Paris, chez Guillaume de Luyne, I694.

ROBERT Raymonde, «Les Conteurs français lecteurs de Basile : $\mathrm{M}^{\text {lle }}$ Lhéritier, $\mathrm{M}^{\text {lle }}$ de La Force, un auteur anonyme, Cazotte", The Romanic Review, vol. 99 (paru en 2010 mais redaté à 2008), nos 3-4, p. 333-348. 